Rev Inv Vet Perú 2018; 29(1): 15-30

http://dx.doi.org/10.15381/rivep.v29i1.14195

Artículo de Revisión

\title{
Requerimientos para la medición de indicadores de estrés invasivos y no invasivos en producción animal
}

\author{
REQUIREMENTS FOR THE MEASUREMENT OF INVASIVE AND NON-INVASIVE STRESS \\ INDICATORS IN ANIMAL PRODUCTION
Rosa Angélica Sanmiguel Plazas ${ }^{1,4}$, Fernely Augusto Plazas Hernández ${ }^{1}$, Dunia Yisela Trujillo Piso ${ }^{1}$, María del Rocío Pérez Rubio" Lina María Peñuela Sierra ${ }^{2}$, Alice DiGiacinto ${ }^{3}$

\section{Resumen}

En esta revisión se presentan los principales requerimientos para medir de manera multimodal, indicadores del bienestar en animales de producción, desde los parámetros productivos hasta los fisiológicos, categorizados como indicadores no invasivos e indicadores invasivos. Se pretende generar una compilación de ideas para que el investigador de acuerdo con las limitaciones, condiciones y alcances de sus recursos evalúe bienestar animal con fines académicos, de investigación y de optimización de la producción y del mercadeo de los productos finales en un sistema de producción pecuario.

Palabras clave: bienestar animal; indicadores de bienestar; multimodal

\section{AbSTRaCT}

This review presents the main requirements for measuring multimodal welfare indicators in production animals, from production parameters to physiological parameters, categorized as noninvasive indicators and invasive indicators. It is intended to generate a collection of ideas so that the researcher, in accordance with the limitations, conditions and scope of its resources, evaluate animal welfare for academic purposes, research and optimization of the production and marketing of the final products in a livestock production system.

Key words: animal welfare; welfare indicators; multimodal

\footnotetext{
${ }^{1}$ Grupo de Investigación IMPRONTA, Universidad Cooperativa de Colombia, Ibagué, Colombia

${ }^{2}$ Grupo de Sistemas Agroforestales Pecuarios, Universidad del Tolima, Ibagué, Colombia

${ }^{3}$ Grupo de Economía Agrícola, Universidad de Wageningen, Wageningen, Holanda

${ }^{4}$ E-mail: rosa.sanmiguel@campusucc.edu.co
}

Recibido: 15 de junio de 2017

Aceptado para publicación: 30 de noviembre de 2017 


\section{INTRODUCCIÓN}

En el ámbito de la producción animal existe una serie de prácticas de manejo e interacciones humano-animal que se requieren para el manejo y la mejora de eficiencia de los animales domésticos, tales como la manipulación, estrategias nutricionales, medicina preventiva, técnicas quirúrgicas, empleo de corrales y jaulas, y mecanismos y medios de transporte, entre otros. Esto puede implicar incomodidad o incluso dolor para los animales, lo cual puede generar estrés y, por consiguiente, cambios comportamentales y fisiológicos que conllevan a la disminución de su bienestar (Mellor et al., 2000; Mpakama et al., 2014).

El estrés es una respuesta inespecífica del organismo animal ante condiciones ambientales adversas que produce ajustes fisiológicos y metabólicos para mantener la homeostasis, genera efectos sobre el sistema nervioso central, el sistema neuroendocrino y el sistema inmune (Sordillo y Aitken, 2008; Feng et al., 2012). En la respuesta neuroendocrina al estrés, el hipotálamo libera el factor liberador de corticotropina, arginina vasopresina, péptidos derivados de la propiomelanocortina, $\beta$ endorfinas y las hormonas catabólicas (catecoloaminas y glucocorticoides) que participan de forma adversa con las hormonas anabólicas (hormona de crecimiento y hormonas gonadales), activando respuestas adaptativas para la sobrevivencia (Charmandari et al., 2005).

Selye (1946) desarrolló un modelo general de respuesta al estrés llamado «Síndrome General y Adaptación» G.A.S. (General Adaptation Syndrome), en donde divide la reacción generada por el organismo sometido a estrés en tres fases: fase de reacción de alarma, citando los cambios fisiológicos y bioquímicos generados por el organismo frente a un estímulo al que no está adaptado cuantitativamente o cualitativamente; fase de resistencia, en la que se resumen todas las reacciones sistémicas expresadas frente a un estímulo prolongado al cual el animal se está adaptando progresivamente; y fase de agotamiento, con la que finalizan las reacciones por la permanencia del estímulo.

Animales con pobre bienestar tienden a sufrir inmunodepresión, disminución de la eficiencia productiva y cambios en el comportamiento (Broom, 2007). Por lo anterior, se puede considerar que diseñar y ejecutar estrategias que incrementen la capacidad de los animales para mitigar los efectos fisiológicos del estrés puede ser importante para mejorar el desempeño productivo. Para ello se requiere evaluar indicadores multimodales que, en conjunto, determinen de manera confiable la severidad del estrés y el cambio en el bienes$\operatorname{tar}$ (Otovic y Hutchinson, 2015).

El tema de bienestar animal no ha sido ajeno a la internacionalización de mercados; de hecho, los países europeos han sido pioneros en llevar a cabo cambios legislativos y de concienciación en favor del bienestar de los animales de producción (Dal mau et al., 2006), incluyendo programas de aseguramiento de la inocuidad alimentaria de origen animal (Gallo, 2008; Mora, 2011). Por ende, es pertinente que la comunidad científica tenga disponibilidad de metodologías para la evaluación del bienestar en los sistemas de producción.

El propósito de la presente revisión es ofrecer una carta de opciones de indicadores de estrés no invasivos e invasivos que permitan elegir, de acuerdo con las posibilidades técnicas y económicas, los más apropiados para evaluar el bienestar de animales de producción sometidos a prácticas requeridas para la optimización de la rentabilidad.

\section{Indicadores No Invasivos}

Los indicadores de estrés no invasivos son datos que facilitan la repetición de muestreos, puesto que para su colecta no afectan de manera prolongada o permanente las libertades de los animales de acuerdo con los conceptos del Farm Animal Welfare 
Council (FAWC, 1979). Esta institución, predecesora del actual Farm Animal Welfare Committee, considera que el estado de bienestar de un animal debe cumplir cinco aspectos o libertades: ausencia de hambre y sed, ausencia de incomodidad física y térmica, ausencia de dolor, lesión o enfermedad, ausencia de miedo y estrés, y capacidad para desplegar la conducta normal de la especie.

Entre los principales indicadores no invasivos (Cuadro 1) se encuentran los siguientes:

\section{Parámetros etológicos}

Condiciones generadoras de estrés promueven respuestas psicoendocrinas (Charmandani et al., 2005), donde los animales afrontan cambios comportamentales, muchas veces estereotipados, inherentes a la amortiguación de la respuesta fisiológica.

El diseño de un protocolo de evaluación de comportamiento y bienestar especie específico genera más confiabilidad, ya que es necesario conocer el comportamiento normal de los animales para identificar los cambios generados por las condiciones en el que el animal se encuentra (Whay et al., 2007; Khalil et al., 2010). En estos estudios se registran patrones de comportamiento tales como acicalamiento, picoteo, agresividad entre congéneres (Dixon y Duncan, 2010; De Jong et al., 2013; Rodenburg et al., 2013), vocalización (Harewood et al., 2005), así como acostarse, levantarse, defecar, orinar (Huynh et al., 2006), jadeo e interacciones agresivas, entre otros. Los resultados se analizan como porcentajes del número de observaciones totales (Candiani et al., 2008), frecuencia de los eventos comportamentales y duración de cada evento (Bulitta et al., 2015). Los datos etológicos son más precisos si se utilizan cámaras de video que registren durante el tiempo del experimento las 24 horas de cada día (Huynh et al., 2006).

\section{Pruebas de miedo}

Considerándose la libertad de miedo entre las cinco libertades del bienestar animal (FAWC, 1979), los test que miden el miedo son indicadores del bienestar (Elrom 2000; Forkman et al., 2007).

El «Arena test» es una prueba usada en mamíferos y para su aplicación se requiere un espacio cerrado cubierto con cama de aserrín en un área adecuada acorde al tamaño del animal, además de cámaras digitales que registren el comportamiento de los animales durante los días de la prueba. Un solo observador debe hacerse responsable del análisis de los videos (Mintline et al., 2012). Esta prueba se utiliza muchas veces para evaluar la reacción de un animal a un objeto extraño.

La inmovilidad tónica (IT) es un estado de parálisis aparente de peces, reptiles, aves y algunos mamíferos como los conejos, como respuesta a una amenaza asociada con cambios en el control simpático vagal (LeitePanissi et al., 2006; Valance et al., 2007). En aves, esta prueba se realiza girando al animal en decúbito dorsal sobre una mesa plana y estable, sosteniéndole el cuerpo y la cabeza durante aproximadamente 15 segundos para contabilizar y registrar el tiempo de inmovilidad que el animal presenta una vez es liberado (Elrom, 2000; Campo y Dávila 2002a,b). Los protocolos de medición de bienestar animal han incluido en los últimos años a la IT como una opción válida de bienestar en aves de producción (Sandoval et al., 2003; Nicol et al., 2009; Sanmiguel et al., 2015). Incluso se ha demostrado que aves que presentan tiempo corto de IT demuestran mejor desempeño en el crecimiento, ganancia de peso, producción de huevo y mayor adaptabilidad al estrés que las aves que presentan un tiempo largo de IT (Castañeda y Gómez, 2010; Wang et al., 2013). 
La evaluación del nivel del miedo durante el desembarque en plantas de faenado de cerdos considera comportamientos como revolverse sobre sí mismo, negarse a avanzar o a retroceder, y avanzar y retroceder en breves lapsos de tiempo (Dalmau et al., 2006).

\section{Parámetros productivos}

Sin duda, son los parámetros más importantes para los productores, puesto que afectan el rendimiento financiero de sus negocios y, a su vez, han sido relacionados con el bienestar de los animales (Beloor et al., 2010).

Es evidente y comprobado por estudios de investigación que el estrés en animales de producción afecta parámetros productivos tales como el consumo de alimento, la ganancia de peso corporal, la producción láctea, la calidad de la masa muscular, y la calidad del huevo (número, peso, grosor de la cáscara, gravedad específica del huevo, y peso y grosor de la albúmina). La toma de datos de parámetros productivos requiere registros ajustados a las variables a medir, equipos de medición, etc., de acuerdo con la especie y tipo de producción (Star et al., 2008; Beloor et al., 2010; Iwama et al., 2011, Sanmiguel y Rondón, 2014).

\section{Parámetros fisiológicos}

\section{- Temperatura corporal, termografia y fre- cuencia respiratoria}

Cambios de temperatura y frecuencia respiratoria pueden ser indicativos de estrés o infección. Para la toma de temperatura corporal es suficiente un termómetro infrarojo para capturar datos de temperatura en espalda, dorso y pierna, en horas específicas del día, de acuerdo con las intenciones de la investigación y teniendo en cuenta el ritmo circadiano del animal. En cerdos, cabras, ovejas y bovinos, la temperatura rectal es un parámetro de uso frecuente para medir bienestar y tan solo se requiere un termómetro digital (Sharma et al., 2013).

La termografía ocular y del dorso también ha sido usada para evaluar estrés en animales de producción. En este caso se requiere una cámara que tome imágenes infrarrojas para su posterior análisis (Stewart et al., 2008; Schmidt et al., 2013).

Para medir la frecuencia respiratoria se necesita contar los movimientos torácicos en el transcurso de un minuto utilizando un cronómetro (Huynh et al., 2006).

\section{- Cortisol en saliva, orina, heces y pelo}

El cortisol que se mide en saliva, orina, heces y pelo son metabolitos de la hormona y no la hormona original; por consiguiente, su estandarización, interpretación y análisis debe ser especie específica (Hernández-Jauregui et al., 2005). Para cualquiera de las muestras a evaluar, es necesario considerar que los kits comerciales para la medición de cortisol y sus metabolitos deben ser validados con la muestra de la especie objeto de estudio, ya sea mediante radioinmunoensayo (Singh, 2009; Otten et al., 2010) o de inmunoabsorbencia ligada a enzimas (ELISA) (Candiani et al., 2008; Carbajal et al., 2014), estableciendo un paralelo entre la curva de dilución de diferentes muestras y la curva estándar previa verificación del coeficiente de variación intraensayo y reactividad cruzada con otros metabolitos como progesterona y testosterona. En este sentido, la medición de cortisol tiene como limitantes los altos costos de los kits comerciales y el requerimiento de equipos especializados, tales como espectrofotómetros de 96 celdas o contadores automatizados para radioinmunoanálisis.

Para la obtención de muestra salival se requieren tubos de recolección de saliva que contienen una barra de metal con una esponja en un extremo o un hisopo de algodón, se- 
gún el manejo de la especie. La determinación de cortisol se realiza mediante un kit de inmunoensayo directo, que tiene un rango detectable de 0 a $100 \mathrm{ng} / \mathrm{ml}$ y una sensibilidad de $0.5 \mathrm{ng} / \mathrm{ml}$ (Harewood y McGowan, 2005; Escribano et al., 2015).

Para la obtención de muestras fecales, dependiendo de la especie, se recogen directamente del recto o de la cama, teniendo en cuenta que no estén contaminadas con orina. Niveles de cortisol en heces son concordantes con cortisol sérico (Sheriff et al., 2010).

En aves, los glucococorticoides se producen como corticosterona (Holmes y Phillips, 1976) y el $75 \%$ es excretado mediante la orina. Por esta razón el muestreo de heces es útil para identificar corticosterona mediante radioinmunoanálisis, posterior a la separación de la porción blanca líquida de las heces aviares (Rettenbacher et al., 2004).

El cortisol en pelo ha sido medido en ovejas como indicador de estrés crónico (Stubsjoen, 2015a). Para realizar el ensayo, se debe almacenar el pelo muestreado en papel aluminio. Se determina la inmunoreactividad de las muestras en un inmunoensayo con enzima de cortisol (Palme y Möstl, 1997; Rettenbacher et al., 2004).

De una manera análoga a la medición de cortisol en pelo de mamíferos, la corticosterona depositada en plumas de las aves es un indicador estable del desafío a estresores que desencadenen actividad del eje hipotálamo hipofisiaria adrenal (Bortolotti et al., 2009; Carbajal et al., 2014). Para la medición de corticosterona en yema o albúmina se requiere pesar el huevo y separar la albúmina de la yema. La corticosterona es determinada con un kit de radioinmunoensayo (Downing y Briden, 2008; Singh et al., 2009; Ahmed et al., 2014).

A la hora de analizar los niveles de cortisol es importante tomar en cuenta cómo se tomó y de donde vino la muestra. No todos los estresores producen un incremento del cortisol. Además, el cortisol, como muchas otras hormonas, tiene un ritmo circadiano, por lo que el momento del muestreo debe ser tomado en cuenta (Möstl y Palme, 2002). Las muestras de pelo son generalmente usadas para medir estrés crónico (Kalra et al., 2007), ya que los cambios en los niveles de cortisol en el pelo no son inmediatos, contrario a lo que ocurre en sangre.

\section{- Test de testosterona salival}

La testosterona salival es reconocida como un indicador de estrés en mamíferos y no es influenciado por el ritmo circadiano; por lo que el muestreo se puede hacer en cualquier hora del día (Escribano et al., 2014). El análisis se realiza mediante una prueba de inmunoensayo enzimático competitivo (Escribano et al., 2014).

\section{- Catecolaminas urinarias}

Concentraciones urinarias de epinefrina, norepinefrina y dopamina son consideradas indicadores de bienestar animal. Una vez colectada la orina, se conserva con $\mathrm{HCl}$ para estabilizar y conservar las catecolaminas urinarias almacenadas a $-70^{\circ} \mathrm{C}$. El análisis e hace mediante un método de fase sólida de intercambio catiónico y determinada mediante cromatografía líquida (O’Neil et al., 2012).

\section{Electrofisiografia}

Las señales bioeléctricas en el organismo animal son consideradas indicadores directos de estrés puesto que cambian respecto a la influencia del sistema nervioso autónomo, dando como consecuencia cambios psicofisiológicos (Berntson et al., 1991), lo cual permite evaluar efectos de estrés por manejo (Hagen et al., 2005), calor (Chacón et al., 2010), desnutrición (Khalil et al., 2010), y emocional (Leliveld et al., 2016), entre otros, generados en las prácticas de producción animal. Incluso, por el efecto de las estaciones del año sobre la actividad simpático vagal cardíaca dependiente del ritmo 
circadiano, especialmente en la época de verano (Kovacs et al., 2016).

Los métodos electrofisiológicos se han diversificado en los últimos años desarrollando equipos de grabación más ligeros y más robustos, por lo que la utilización se ha incrementado tanto en diagnóstico como en investigación (Chorev et al., 2009). No obstante, el alto costo del equipamiento limita su uso. Entre las principales señales bioélectricas se encuentran las registradas a partir de un electrocardiógrafo con electrodos desechables o reutilizables de superficie. Los registros electrocardiográficos (ECG) se determinan para evaluar la variación de amplitud y duración de ondas (Cinar et al., 2006), segmentos, intervalos, ritmo, frecuencia cardíaca y variabilidad de la frecuencia cardíaca; tanto en el dominio del tiempo como en el dominio de la frecuencia (Kovacs et al., 2015; Leliveld et al., 2016).

En grandes animales (bovinos, equinos y cerdos) se utiliza un cinturón con dos electrodos integrados y un transmisor específico que presenta los registros ECG en un data logger (Kovacs et al., 2015; Leliveld et al., 2016). Los electrodos se fijan sobre la piel con gel transmisor de ultrasonidos en el sitio de cada electrodo (Frondelius et al., 2015). En aves domésticas se utiliza una unidad telemétrica remota con la ubicación de electrodos adhesivos en la quilla, asegurados con un arnés que no restringe el movimiento (Davies et al., 2014).

La mayoría de los equipos traen plantillas estandarizadas con filtros y velocidad para mamíferos; no obstante, por las características específicas de la actividad ECG en aves, la plantilla de registro debe estandarizarse a $10 \mathrm{~mm}=1 \mathrm{mV}$ y a una velocidad de $50 \mathrm{~mm} / \mathrm{s}$, teniendo en cuenta que las derivadas significativas para su lectura e interpretación son II, III y aVR (Hassanpour et al., 2008).

\section{- Variabilidad de la frecuencia cardíaca (VFC)}

La medición de la variabilidad de la frecuencia cardíaca (HRV en inglés) mide el equilibrio de la actividad del sistema nervioso autónomo. En las últimas décadas, la VFC ha tomado fuerza en la investigación en bienestar animal para analizar cambios en el equilibrio simpático-vagal tanto en mamíferos como en aves, en relación con el ritmo circadiano (Brauner et al., 2010), manejo, salud, factores de estrés psicológico y ambientales (Stubjoens et al., 2015b); así como para analizar características genéticas tales como respuestas comportamentales, temperamento y estado de ánimo (Borell et al., 2007; Leliveld et al., 2016).

A partir de un registro electrocardiográfico, mediante un software comercial, se puede obtener datos del intervalo R-R (o $\mathrm{S}-\mathrm{S}$ en el caso de las aves) para analizarlos en el dominio del tiempo o en el dominio de la frecuencia. El análisis de la VFC en el dominio del tiempo incluye el promedio de tiempo de intervalo entre latidos R-R, la desviación estándar del intervalo ente latidos y la raíz cuadrada de la media de las diferencias sucesivas. Valores altos de esta última indica actividad vagal significativa.

El análisis de la VFC en el dominio de la frecuencia es el espectro obtenido mediante Fast Fourrier Transform (FFT); determinado mediante un software especializado, donde la baja frecuencia (BF) es asociada con actividad simpática y la alta frecuencia (AF) representa actividad parasimpática (Migliaro et al., 2004). Un incremento en la relación $\mathrm{BF} / \mathrm{AF}$ es interpretada como una dominancia simpática y viceversa, lo cual se da cuando hay emociones como enojo y hostilidad (Borell et al., 2007; Tarvainen et al., 2014). 


\section{- Electroencefalografía}

E1 EEG representa el voltaje entre dos electrodos ubicados sobre la piel del cráneo o implantado quirúrgicamente (Purves et al., 2001). En bienestar animal es usado para medir el nivel de dolor y nivel de conciencia e inconciencia en diferentes actividades tales como la eutanasia, analgesia y anestesia (Benson et al., 2012), así como el efecto de sonidos y periodos de hambre (Cwynar y Zawadzki, 2010; Otto et al., 2012).

Para su medición se requiere un electroencefalógrafo con electrodos soldados de manera permanente a los cables y un sistema de mínimo cuatro canales de datos de prospección, vinculados a un software para la grabación, reproducción y análisis de los registros (Cwynar y Zawadzki, 2010). Los registros EEG se analizan de acuerdo con los patrones EEG determinados mediante la relación alfa/delta (Gibson et al., 2007).

\section{Indicadores Invasivos}

Los indicadores invasivos requieren manipulación especial de los animales sometidos al experimento que generan algún tipo de incomodidad o dolor como la extracción de sangre, uso de electrodos de aguja, cateterización, cirugías y biopsias, entre otros. Por esta razón se debe tener en cuenta que los datos obtenidos están sujetos a los cambios fisiológicos que generan el estrés agudo en la colecta de muestras y la manipulación de los individuos. Por otro lado, en los métodos invasivos, los animales deben ser restringidos y manipulados, lo que puede generar estrés per se.

Calidad de canal possacrificio: metodología para su evaluación

La calidad de la carne se define por sus propiedades fisicoquímicas $(\mathrm{pH}$, capacidad de retención de agua, color, textura, etc.), organolépticas (suavidad, consistencia, olor, sabor, etc.) y bacteriológicas, que dependen de muchos factores, incluido el estrés. Un deficiente manejo ante mortem impacta negativamente en los atributos de la carne a consecuencia de los cambios hormonales que afectan la composición del tejido muscular en el animal en vivo y las consecuentes características de la carne obtenida. Tanto el manejo ante mortem como el método de insensibilización durante el sacrificio afecta su calidad (Hernández et al., 2013).

El beneficio de animales incluye movimientos previos al sacrificio que inducen al estrés, lo que afecta la calidad de la canal. El $\mathrm{pH}$ normal del músculo es de 7.1 y el de la carne debe ser de 5.4 a 5.7. Esto se da porque las reservas de glucógeno muscular se convierten en ácido láctico, lo cual disminuye el pH de la carne. Las reservas de glucógeno en el animal pueden disminuir rápidamente por factores de estrés y ejercicio excesivo, de allí que pH mayores a 5.7 en la carne se relacionan a un manejo inadecuado de los animales, como maltrato y transporte por más de 10 horas (Toledo y Vargas, 2016).

En bovinos, ovinos, pavos y cerdos se puede presentar canales DFD (por sus siglas en inglés, oscuras, secas y firmes) de baja calidad con $\mathrm{pH}$ de 6.4-6.8, lo que afecta el tiempo de uso de la carne (Chambers y Gradin, 2001). El efecto del estrés en bovinos antes del sacrificio se manifiesta con $\mathrm{pH}$ por encima de 6.2 (Hernández et al., 2013). Por otra parte, los ovinos son menos sensibles al estrés que los bovinos y porcinos y, por tanto, menos susceptibles a problemas de $\mathrm{pH}$ en su canal; sin embargo, pueden presentar $\mathrm{pH}$ mayores de 6 cuando son sometidos a estrés (Sañudo y González, 2008).

El estrés también puede producir carnes de tipo PSE (pálida, suave y exudativa), carnes con un bajo $\mathrm{pH}$ probablemente debido a un incremento de la acidosis metabólica. Un alto porcentaje de los cerdos a nivel mundial desarrollan carnes PSE, pero este porcentaje varía dependiendo del manejo (Adzitev y Nuruk, 2011; Vermeulen et al., 2015). 
Por lo anterior, la medición del pH a los 45 min y 24 h que se emplea para tipificar la calidad de la carne (PSE y DFD) se puede utilizar para determinar el nivel de estrés ante mortem. El pH se puede medir a los 45 min y a las $24 \mathrm{~h}$ del sacrificio mediante el uso de un pHmetro con sonda de silicio calibrado con tampones $\mathrm{pH} 4$ y $\mathrm{pH} 7$ (recalibrando cada 10 lecturas), en el músculo semimebranoso en porcinos y largo del dorso en bovinos (Jara, 2007). Se consideran canales con PSE aquellas que a los 45 min tengan un $\mathrm{pH} \leq 5.8 \mathrm{y}$ aquellas con valores $\geq 6.3$ se consideran DFD. Del mismo modo, canales que a las $24 \mathrm{~h}$ tengan $\mathrm{pH}$ de $<5.6$ se consideran PSE y aquellas con $\mathrm{pH} \geq 6.2$ se consideran DFD. En consecuencia, las carnes con PSE se consideran como indicativo de animales con predisposición al estrés y sometidos a factores estresantes (Castrillón et al., 2007).

\section{Parámetros hematológicos}

Para la medición de parámetros sanguíneos se requiere tomar muestras sanguíneas por venipuntura en un tubo con anticoagulante (heparina para química sanguínea y EDTA para hematología) y sin anticoagulante para serología (Dacie y Lewis 1991).

\section{- El hematocrito}

Como consecuencia de la contracción esplénica provocada por la liberación de catecolaminas frente a un estresor agudo, el hematocrito es afectado, tanto en mamíferos como en aves y peces (Wang et al., 2004). Para valorar el hematocrito se llena un microcapilar de la sangre colectada con EDTA, se centrifuga a $2000 \mathrm{~g}$ durante $5 \mathrm{~min}$ $\mathrm{y}$, posteriormente, se realiza la lectura en una regla lectora de microhematocrito (Campbell, 2004).

\section{- La relación neutrófilos/linfocitos (N/L)}

Esta relación es considerada un indicativo indirecto de niveles de estrés, puesto que el incremento en los niveles elevados de cortisol estimula la producción de neutrófilos inmaduros de la médula ósea a la sangre periférica y disminución de linfocitos (Akram et al., 2002; Mashaly et al., 2004; Ajakaiye et al., 2010). Esto se refleja en el incremento de la relación $\mathrm{N} / \mathrm{L}$ en presencia de factores predisponentes de estrés (Davis et al., 2000; Shini et al., 2008). En aves, la analogía se hace con la relación heterófilos/linfocitos $(\mathrm{H} /$ L) bajo los mismos conceptos fisiológicos mencionados para mamíferos (Rath et al., 2006; Singh, 2009).

Para medir la relación N/L se requieren $50 \mu 1$ de sangre con anticoagulante, se hace un extendido en lámina portaobjetos seguido de tinción sanguínea y posterior observación mediante un microscopio de luz, en el cual se realiza una lectura de cantidad de neutrófilos (heterófilos en aves) y se divide por la cantidad de linfocitos en un conteo total de 100 células sanguíneas por muestra (Rath et al., 2006).

\section{Química sanguínea}

\section{- Proteínas de fase aguda}

La medición de proteínas de fase aguda es calculada como un porcentaje del contenido de proteínas totales de cada muestra. Así, es necesario determinar la concentración de proteínas séricas totales con un kit comercial de colorimetría y cuantificación basado en el ácido bicinconínico. Las proteínas de fase aguda se determinan mediante un kit comercial de electroforesis para detectar albúmina, basado en la separación electroforética de proteínas en un gel agarosa tamponado (Candiani et al., 2008). Estos métodos requieren la disposición de equipos electroforéticos, densitómetros y espectrofotómetros.

\section{- Creatinakinasa}

La creatinakinasa se determina mediante suero sanguíneo conservado en refrigeración entre 2 y $10{ }^{\circ} \mathrm{C}$, con el uso un kit comercial de inmunoinhibición en el que por técnica de espectrofotometría se desarrolla una prue- 
ba liquida UV activada por NAC (Mitchell et al., 1998; Ceballos et al., 2013). También existen kits comerciales para la identificación de la creatinakinasa por nefelometría (Bulitta et al., 2015).

\section{- Cortisol sérico}

Para evaluar el cortisol sérico (o corticosterona sérica en aves) se requiere una muestra de suero sanguíneo y un kit de radioinmunoensayo competitivo (Otten et al., 2010; Bulitta et al., 2015), de ELISA (Dehnhard et al., 2002; Candiani et al., 2008; Ahmed et al., 2014) o de quimioluminiscencia (Ceballos et al., 2013).

\section{Respuesta inmune humoral}

Para medir títulos de anticuerpos se requiere suero sanguíneo y el uso de kits comerciales de inhibición de hemaglutinación, inmunoabsorbancia u otras técnicas de microtitulación de acuerdo con el tipo de anticuerpo que se pretenda medir (Sandhu et al., 2007; Ghareeb et al., 2012).

\section{Telemetría}

Mediante registros telemétricos se pueden obtener datos de frecuencia cardiaca, variabilidad de la frecuencia cardíaca, temperatura corporal y presión sanguínea, entre otros. Se implanta quirúrgicamente un transmisor telemétrico de ubicación intraperitoneal en mamíferos o en la quilla de las aves, con los electrodos fijados subcutáneamente en diferentes sitios de acuerdo con la especie (Valance et al., 2007; Kostelanets et al., 2009; Khalil et al., 2010). Los altos costos de los sensores telemétricos y demás equipamiento limitan la aplicación de esta técnica.

Expresión de genes

La medición de la expresión de genes ha contribuido de manera significativa en la comprensión de los procesos homeostáticos del organismo y el efecto de los factores re- lacionados con el estrés. La muestra de sangre u otros tejidos representativos como hígado, bazo o yeyuno de donde se va a colectar el ARN, debe ser congelado entre $-70 \mathrm{y}$ $-80^{\circ} \mathrm{C}$ o en nitrógeno líquido hasta el momento del análisis (Beloor et al., 2010; Kang et al., 2011).

Esta metodología requiere el uso del equipamiento para RT-PCR semi quantitativo o RT-PCR quantitativo en tiempo real tales como homogenizador, centrifuga, detector fluorescente, espectrofotómetro, incubadora, termociclador, densitómetro y el kit comercial del gen objeto de estudio y sus correspondientes cebadores (Hangalapura et al., 2006; Kang et al., 2011; Feng et al., 2012).

\section{Biomarcadores de estrés oxidativo}

El estrés oxidativo es causado por radicales libres, especies reactivas al oxígeno, que generan daños en el DNA, lípidos de la membrana celular, proteínas y otras macroléculas, contribuyendo al detrimento de la salud y parámetros productivos (Sordillo y Aitken, 2008; Lu et al., 2010). Una manera de medir el proceso de estallido respiratorio de los fagocitos en sangre entera es mediante espectrofotometría (Mohanty y Sahoo, 2010).

También se puede emplear kits comerciales espectrofotométricos para medir la actividad de enzimas que participan en el estrés oxidativo, tales como la glutatión peroxidasa sérica, catalasa sérica, glutatión transferasa y superóxido dismutasa (Ismail et al., 2013).

\section{Seguimiento de la Presentación de En- fermedades Asociadas al Estrés}

De acuerdo con el Welfare Quality Protocol (2009), uno de los parámetros a tener en cuenta para medir el bienestar de un sistema de producción animal es el seguimiento de la presentación de enfermedades asociadas al estrés (Forkman y Keeling, 2009). Por ejemplo, en avicultura la privación de movimiento conduce a la presentación de 
osteoporosis y, por consiguiente, fracturas. Asimismo, una presentación pobre de la cama genera pododermatitis (Webster, 2003; Sans et al., 2014). De la misma manera, en mamíferos son reconocidos los higromas, prolapsos uterinos y problemas en la salud podal como enfermedades asociadas al estrés (De Rosa et al., 2015; Guccione et al., 2016).

\section{Literatura Citada}

1. Adzitey F, Nurul H. 2011. Pale soft exudative (PSE) and dark firm dry (DFD) meats: causes and measures to reduce these incidences-a mini review. Int Food Res J 18: 11-20.

2. Ahmed A, Ma W, Ni Y, Wang S, Zhao R. 2014. Corticosterone in ovo modifies aggressive behaviors and reproductive performances through alterations of the hipotalamic-pituitary-gonadal axis in the chicken. Anim Reprod Sci 146: 193-201. doi: 10.1016/j.anireprosci.2014.02.013

3. Ajakaiye J, Ayo J, Ojo S. 2010. Effect of heat stress on some blood parameters and egg production of Shika Brown layer chickens transported by road. Biol Res 43: 183-189. doi: /S0716-97602010000200006

4. Akram M, Zia-ur-rahman C, Kim S. 2002. Effect on the induced molting on the relative weights and hormone levels of thyroid, ovary and adrenal glands in spent laying hens. Korean J Poult Sci 29: 243-247.

5. Berntson GG, Cacioppo JT, Quigley KS. 1991. Autonomic determinism: the modes of autonomic control, the doctrine of autonomic space, and the laws of autonomic constraint. Psychol Rev 98: 459-487.

6. Borell E, Langbein J, Deprés G, Hansen S, Leterrier C, MarchantForder J, Marchant-Forder R, et al. 2007. Heart rate variability as a measure of autonomic regulation of cardiac activity for assessing stress and welfare in farm animals - a review. Physiol
Behav 92: 293-316. doi: 10.1016/ j.physbeh.2007.01.007

7. Bortolotti G, Marchant T, Blas J, Cabezas S. 2009. Tracking stress: localization, deposition and stability of corticosterone in feathers. J Exp Biol 212: 1477-1482. doi: 10.1242/jeb.022152

8. Beloor J, Kang H, Kim Y, Subramani V, Jang I, Sohn S, Moon Y. 2010. The effect of stocking density on stress related genes and telomeric length in broiler chickens. Asian Aust J Anim Sci 23: 437-443. doi: 10.5713/ajas.2010.90400

9. Benson E, Alphin R, Rankin M, Caputo M, Kinney C, Johnson A. 2012. Evaluation of EEG based determination of unconsciousness vs. loss of posture in broilers. Res Vet Sci 93: 960-964. doi: 10.1016/j.rvsc.2011.12.008

10. Brauner A, Kurjiaka D, Ibragimov A, Baldwin A. 2010. Impact of cage size and enrichment (tube and shelf) on heart rate variability in rats. Scand J Lab Anim Sci37: 185-201.

11. Broom D. 2007. Welfare in relation to feelings, stress and health. REDVET 8: 12B. [Internet]. Available in: http:// www.veterinaria.org/revistas/redvet/ n121207B/BA018ing.pdf

12. Bulitta F, Aradom S, Gebresenbet $G$ 2015. Effect of transport time of up to 12 hours on welfare of cows and bulls. J Service Sci Manag 8: 161-182. doi: 10.4236/jssm.2015.82019

13. Carbajal A, Tallo-Parra O, SabesAlsina M, Mular I, López-Bejar M. 2014. Feather corticosterone evaluated by ELISA in broilers: a potential tool to evaluate broiler welfare. Poult Sc 93: 2884-2886. doi: 10.3382/ps.2014-04092

14. Campbell T. 2004. Hematology of birds. In: Thrall MA (ed). Veterinary hematology and clinical chemistry. Philadelphia: Lippincott Williams and Wilkins. p 225-258.

15. Campo JL, Dávila SG. $2002 a$. Changes in heterophil to lymphocyte ratios of heat-stressed chickens in 
response to dietary supplementation of several related stress agents. Arch Geflügelk 66: 80-84.

16. Campo JL, Dávila SG 2002b. Effect of photoperiod on heterophil to lymphocyte ratio and tonic immobility duration of chickens. Poult Sci 81: 1637-1639.

17. Candiani D, Salamano G, Mellia E, Doglione L, Bruno R, Thoussaint M, Gruys E. 2008. A combination of behavioral and physiological indicators for assessing pig welfare on the farm. J Appl Anim Welf Sci 11: 1-13. doi: 10.1080/ 10888700701729080

18. Ceballos D, Villa M, Zimerman M, Opazo W, Tracaman J. 2013. Evaluación de canales e indicadores fisiológicos de estrés en ovejas merino de refugo faenadas a diferente tiempo post esquila. Instituto Nacional de Tecnología Agropecuaria. [Internet]. Disponible en: https://inta.gob.ar/sites/default/files/ script-tmp-inta-evaluacion-de-canales-eindicadores-fisiologicos.pdf

19. Castañeda C, Gómez J. 2010. Evaluación del bienestar animal y comparación de los parámetros productivos en gallinas ponedoras de la línea Hy-line Brown en tres modelos de producción: piso, jaula y pastoreo. Rev Cienc Anim 3: 9-22.

20. Castrillón W, Fernández J, Restrepo L. 2007. Variables asociadas con la presentación de carne PSE (pálida, suave, exudativa) en canales de cerdo. Rev Colom Cienc Pecua 20: 327-338.

21. Chacon T, Comerma-Steffensen S, Colina Y, Rojas J, Rossini M, Zerpa H, Oliveros I, et al. 2010. Frecuencia cardíaca como indicador de estrés calórico en pollos de engorde. Zootecnia Trop 28: 93-100.

22. Chambers PG, Gradin T. 2001. Directrices para el manejo, transporte y sacrificio humanitario del ganado. Roma: FAO. [Internet]. Disponible en: http:// www.fao.org/docrep/005/x6909S/ x6909S00.HTM

23. Charmandari E, Tsigos C, Chrousos $G$ 2005. Endocrinology of the stress responses. Annu Rev Physiol 67: 259-
284. doi: 10.1146/annurev.physiol.67.040403.120816

24. Chorev E, Epsztein J, Houweling A, Lee A, Brecht M. 2009. Electrophysiological recordings from behaving animals - going beyond spikes. Curr Opin Neurobiol 19: 513-519. doi: 10.1016/ j.conb.2009.08.005

25. Çýnar A, Belge F, Donmez N, Tap D, Selçuk M, Tatar M. 2006. Effects of stress produced by adrenocorticotropin (ACTH) on ECG and some blood parameters in vitamin $\mathrm{C}$ treated and nontreated chickens. Veterinarski Arhiv 76: 227-235.

26. Cwynar P, Zawadzki W. 2010. Recording of bioelectrical activity changes in sheep cerebral cortex. Arch Med Vet 42: 51-62.

27. De Rosa G, Grasso F, Winckler C, Bilancione A, Pacelli C, Masucci F, Napolitano F. 2015. Application of the Welfare Quality protocol to dairy buffalo farms: prevalence and reliability of selected measures. J Dairy Sci 98: 68866896. doi: 10.3168/jds.2015-9350

28. Dacie J, Lewis S. 1991. Practical haematology. $7^{\text {th }}$ ed. London: Churchill Livinstone. p 659-661.

29. Dalmau A, Rodríguez, Pelarde A. 2006. Valoración del bienestar animal del cerdo. Parámetros evaluados en el matadero. Eurocarne 151: 47-56.

30. Davis G, Anderson K, Carroll A. 2000. The effects on long-term caging and molt of single comb of Single Chite White Leghorn hens on heterophil to lymphocyte ratios, corticosterone and thyroid hormones. Poult Sci 79: 514-518.

31. Davies A, Radford A, Nicol C. 2014. Behavioural and physiological expression of arousal during decision-making in laying hens. Physiol Behav 123: 93-99. doi: 10.1016/j.physbeh.2013.10.008

32. Dehnhard M, Schreer A, Krone O, Jewgenow K, Krause M, Grossmann R. 2002. Measurement of plasma corticosterone and fecal glucocorticoid metabolites in the chicken (Gallus domesticus), the great cormorant 
(Phalacrocorax carbo), and the goshawk (Accipiter gentilis). General Comp Endocrinol 131: 345-352. doi: 10.1016/S0016-6480(03)00033-9

33. De Jong I, Gunnink H, Rommers, M, Bracke, M. 2013. Effect of substrate during early rearing on floor- and feather pecking behaviour in young and adult laying hens. Arch Geflugelk 77: 15-22.

34. Dixon L, Duncan I. 2010. Changes in substrate access did not affect early feather-pecking behavior in two strains of laying hen chicks. J Appl Anim Welf Sci 13: 1-14. doi: 10.1080/10888700903369248

35. Downing J, Bryden W. 2008. Determination of corticosterone concentra-tions in egg albumen: a non-invasive indicator of stress in laying hens. Physiol Behav 95: 381-387. doi: 10.1016/j.physbeh.2008.07.001

36. Escribano D, Gutierrez A, Teccles F, Cerón J. 2015. Changes in saliva biomarkers of stress and immunity in domestic pigs exposed to a psychosocial stressor. Res Vet Sci 102: 38-44. doi: 10.1016/j.rvsc.2015.07.013

37. Escribano D, Fuentes-Rubio M, Cerón J. 2014. Salivary testosterone measurements in growing pigs: validation of an automated chemiluminescent immunoassay and its possible use as an acute stress marker. Res Vet Sci 97: 2025. doi: 10.1016/j.rvsc.2014.04.001

38. Elrom K. 2000. Handling and transportation of broilers - welfare, stress, fear and meat quality. Part III. Fear; definitions, its relation to stress, causes of fear, responses of fear and measurement of fear. Israel J Vet Med 55: 3 .

39. Farm Animal Welfare Council. 1979. Five freedoms. [Internet]. Available in: ht t p://webarchive.nationalarchives.gov.uk/20121010012427/http:// www.fawc.org.uk/freedoms.htm

40. Feng $Y$, Yang $X$, Wang $Y$, Li W, Liu W, Yin R, Yao J. 2012. Effects of immune stress on performance parameters, intestinal enzyme activity and mRNA expression of intestinal transporters in broiler chickens. Asian Austral J Anim Sci 25: 701-707. doi: 10.5713/ajas.2011.11377

41. Forkman B, Boissy A, MeunierSalaün M, Canali E, Jones R. 2007. A critical review of fear tests used on cattle, pigs, sheep, poultry and horses. Physiol Behav 9: 340-374. doi: 10.1016/ j.physbeh.2007.03.016

42. Forkman B, Keeling L. 2009. Assessment of animal welfare measures for layers and broilers. Welfare Quality Reports 9. UK: Cardiff Uniersity. 176 p.

43. Frondelius L, Jarvenranta K, Koponen T, Mononen J. 2015. The effects of body posture and temperament on heart rate variability in dairy cows. Physiol Behav 139: 437-441. doi: 10.1016/j.physbeh.2014.12.002

44. Gallo C. 2008. Using scientific evidence to inform public policy on the long distance transportation of animals in South America. Vet Ital 44: 113-120.

45. Ghareeb K, Awad W, Bohm J. 2012. Ameliorative effect of a microbial feed additive on infectious bronchitis virus antibody titer and stress index in broiler chicks fed deoxinivalenol. Poult Sci 91: 800-807. doi: 10.3382/ps.2011-01741

46. Gibson T, Johnson C, Stafford K, Mitchinson S, Mellor D. 2007. Validation of the acute electroencephalographic responses of calves to noxious stimulus with scoop dehorning. N Z Vet J 55: 152-157. doi: 10.1080/00480169.2007.36760

47. Guccione J, Carcasole C, Alsaaod M, D'Andrea L, Di Loria A, De Rosa A, Ciaramella P, Steiner A. 2016. Assessment of foot health and animal welfare: clinical findings in 229 dairy Mediterranean buffaloes (Bubalus bubalis) affected by foot disorders. BMC Vet Res 12: 1-9. doi: 10.1186/ s12917-016-0726-4

48. Hagen K, Langbein J, Schmied C, Lexer D, Waiblinger S. 2005. Heart rate variability in diary cows-influences of breed and milking system. Physiol 
Behav 85: 195-204. doi: 10.1016/ j.physbeh.-2005.03.019

49. Hangalapura $B$, Kaiser $M$, van der Poel JJ, Parmentier H, Lamont $S$. 2006. Cold stress equally enhances in vivo pro-inflammatory cytokine gene expression in chicken lines divergently selected for antibody responses. Dev Comp Immunol 30: 503-511. doi: 10.1016/j.dci.2005.07.001

50. Hassanpour H, Zamani A, Teshfam M, Zarei H. 2008. Effect of ascorbic acid on the electrocardiogram of broiler chickens raised at high altitude. Nigerian Vet J 29(2): 8-14.

51. Harewood E, McGowan C. 2005. Behavorial and physiological responses to stabling in naive horses. J Equine Vet Sci 25: 164-170. doi: 10.1016/ j.jevs.2005.03.008

52. Hernández-Jauregui D, Galindo F, Valdés R, Romano M, Schuneman A. 2005. Cortisol en saliva, orina y heces: evaluación no invasiva en mamíferos silvestres. Vet Mex 36: 325-337.

53. Hernández J, Aquino J, Rios F. 2013. Efecto del manejo pre-mortem en la calidad de la carne. Necameh 7(2): 41-64.

54. Holmes WN, Phillips JG. 1976. The adrenal cortex of birds. In: ChesterJones I, Henderson IW (eds). General, comparative and clinical endocrinology of the adrenal cortex. London: Academic Press. p 293-420.

55. Huynh T, Aarnink A, Truong C, Kemp $B$, Verstegen M. 2006. Effects of tropical climate and water cooling methods on growing pig's responses. Livestock Sci 104: 278-291. doi: 10.1016/ j.livsci.2006.04.029

56. Iwama GK, Pickering AD, Sumpter JP, Schreck CB. 2011. Fish stress and health in aquaculture. UK: Cambridge University Press. 278 p.

57. Ismail IB, Al-Busadah KA, El-Bahr SM. 2013. Oxidative stress biomarkers and biochemical profile in broilers chicken fed zinc bacitracin and ascorbic acid under hot climate. Am J Biochem Mol Biol 3: 202-214. doi: 10.3923/ajbmb.2013.202.214
58. Jara P. 2007. Efecto del $\mathrm{pH}$ sobre la conservación de carne de bovino de corte oscuro (DFD) envasada al vacío, almacenada a $0{ }^{\circ} \mathrm{C}$. Tesis de grado. Valdivia, Chile: Univ. Austral de Chile. $91 \mathrm{p}$.

59. Kang S, Ko Y, Moon Y, Sohn S, Jang I. 2011. Effect of the combined stress induced by stocking density and feed restriction on hematological and cytokine parameters as stress indicators in laying hens. Asian Austral J Anim Sci 24: 414420. doi: 10.5713/ajas.2011.10315

60. Kalra S, Einarson A, Karaskov T, Van Uum S, Koren G 2007. The relationship between stress and hair cortisol in healthy pregnant women. Clin Invest Med 30: 103-107.

61. Khalil A, Matsui K, Takeda K. 2010. Responses to abrupt changes in feeding and illumination in laying hens. Turk J Vet Anim Sci 34: 433-439. doi: 10.3906/vet0901-25

62. Kostelanetz S, Dickens M, Romero M. 2009. Combined effects of molt and chronic stress on heart rate, heart rate variability and glucocorticoid physiology in European starlings. Comp Biochem Physiol Mol Integr Physiol 154: 493-501. doi: 10.1016/j.cbpa.2009.08.005

63. Kovacs L, Kézer F, Toszer J, Szenci O, Poti P, Pajor F. 2015. Heart rate and heart rate variability in dairy cows with different temperament and behavioural reactivity to humans. PLos One 10(8): e0136294. doi: 10.1371/ journal.pone.0136294

64. Kovács L, Kézér F, Ruff F, Szenci O. 2016. Cardiac autonomic activity has a circadian rhythm in summer but not in winter in non-lactating pregnant dairy cows. Physiol Behav 155: 56-65. doi: 10.1016/j.physbeh.2015.11.031

65. Leliveld L, Dupjan S, Tuchscherer A, Puppe B. 2016. Behavoioural and physiological measures indicate subtle variations in the emotional valence of young pigs. Physiol Behav 157: 116-124. doi: 10.1016/j.physbeh.2016.02.002 
66. Leite-Panissi, CR, Ferrarese, AA, Terzian, $A L$, Menescal-de-Oliveira $L$. 2006. Serotoninergic activation of the basolateral amygdala and modulation of tonic immobility in guinea pig. Brain Res Bull 69: 356-364. doi: 10.1016/ j.brainresbull.2006.02.007

67. Lu T, Piao XL, Zhang $Q$, Wang D, Piao XS, King SW. 2010. Protective effects of Forsythia suspensa extract against oxidative stress induced by diquat in rats. Food Chem Toxicol 48: 764-770. doi: 10.1016/j.fct.2009.12.018

68. Mashaly M, Hendricks G, Kalama M, Gehad A, Abbas A, Patterson P. 2004. Effect of heat stress on production parameters and immune responses of commercial laying hens. Poult Sci 83: 889-894.

69. Mellor DJ, Cook, CJ, Stafford KJ. 2000. Quantifying some responses to pain as a stressor. In: Moberg GP, Mench JA(eds). The biology of animal stress: basic principles and implications for animal welfare. UK: CABI Publishing. p 171-198.

70. Migliaro, E, Canetti, $R$, Contreras $P$, Hakas M, Eirea G, Machado A. 2004. Procesamiento de señales para el estudio de la variabilidad de la frecuencia cardiaca. En: Armentano R, D'Attellis C, Risk M, Vera de Payer E (eds). Procesamiento de señales e imágenes: teoría y aplicaciones. Buenos Aires, Argentina: Universidad Tecnológica Nacional. p 189-204.

71. Mintline E, Woodb S, Passillé A, Rushenb J, Tucker C. 2012. Assessing calf play behavior in an arena test. Appl Anim Behav Sci 141: 101-107. doi: 10.1016/j.applanim.2012.08.006

72. Mitchell M, Kettlewell P. 1998. Physiological stress and welfare of broiler chickens in transit: solutions not problems! Poult Sci 77: 1803-1814.

73. Mohanty B, Sahoo P. 2010. Immune responses and expression profiles of some immune-related genes in Indian major carp, Labeo rohita to Edwardsiella tarda infection. Fish Shellfish Immunol 28: 613-621. doi: 10.1016/ j.fsi.2009.12.025
74. Möstl E, Palme R. 2002. Hormones as indicators of stress. Domest Anim Endocrinol 23: 67-74. doi: 10.1016/ S0739-7240(02)00146-7

75. Mpakama T, Chulayo A, Muchenje V. 2014. Bruising in slaughter cattle and its relationship with creatine kinase levels and beef quality as affected by animal related factors. Asian Austral J Anim Sci 27: 717-725. doi: 10.5713/ajas.2013.13483

76. Mora A. 2011 Enfoque eficiente del bienestar animal en el contexto nacional e internacional. Rev Colomb Cienc Pecu 24: 327-331.

77. Nicol C, Caplen G, Edgar J, Browne W. 2009. Association between welfare indicators and environmental choice in laying hens. Anim Behav 78: 413-424. doi: 10.1016/j.anbehav.2009.05.016

78. O'Neil H, Webb E, Frylinck L, Strydom P. 2012. Urinary catecholamine concentrations in three beef breeds at slaughter. South Afr J Anim Sci 42: 545-549.

79. Otovic P, Hutchinson E. 2015. Limits to using HPA axis activity as an indication of animal welfare. Altex 32: 41-50. doi: 10.14573/altex.1406161

80. Otten W, Kanitz E, Couret D, Veissier I, Prunier A, Merlot E. 2010. Maternal social stress during late pregnancy affects hypothalamic-pituitary-adrenal function and brain neurotransmitter systems in pig offspring, Domest Anim Endocrinol 38: 146-156. doi: 10.1016/ j.domaniend.2009.09.002

81. Otto K, Cebotari S, Hoffler $\mathrm{H}$, Tudorache I. 2012. Electroencephalographic Narcotrend index, spectral edge frequency and median power frequency as guide to anesthetic depth for cardiac surgery in laboratory sheep. Vet J 191: 354-359. doi: 10.1016/ j.tvj1.2011.02.023

82. Palme R, Möstl E. 1997. Measurement of cortisol metabolites in faeces of sheepas a parameter of cortisol concentration in blood. Z Saugetierkd 62: 192-197. 
83. Purves D, Augustine GJ, Fitzpatrick D, Katz L, Lamanta AS, McNamara, JO Williams, SM. 2001. Neuroscience. $2^{\text {nd }}$ ed. Sunderland, MA, USA: Sinauer Associates. $681 \mathrm{p}$.

84. Rath N, Huff W, Huff G 2006. Effects of humic acid on broiler chickens. Poult Sci 85: 410-414.

85. Rettenbacher S, Möstl E, Hackl R, Ghareeb K, Palme R. 2004. Measurement of corticosterone metabolites in chicken droppings. Br Poult Sci 45: 704711. doi: 10.1080/00071660400006156

86. Rodenburg $T$, van Krimpen $M$, De Jong I, De Haas E, Kops M, Riedstra $B$, et al. 2013. The prevention and control of feather pecking in laying hens: identifying the underlying principles. Worlds Poult Sci J 69: 361-374.

87. Sandoval G, Terraes J, Revidatti F. Fernández R, Gauna C, Martín G. 2003. Hematocrito, relación heterófilo linfocito e inmovilidad tónica en pollos con estrés psico-físico crónico criados en jaulas. Com Cient Tecnol V-026. [Internet]. Disponible en: http://www.unne.edu.ar/ unnevieja/Web/cyt/cyt/2003/comunicaciones/04-Veterinarias/V-026.pdf

88. Sandhu M, Rahman Z, Rahman S. 2007. Effects of induced molting on some immunological parameters in laying hens (Gallus domesticus). Arch Geflügelk 71: 110-116.

89. Sañudo A, González C. 2008. Aspectos estratégicos para obtener carne ovina de calidad en el Cono Sur americano. Buenos Aires, Argentina: Agencia Española de Cooperación Internacional (AECI). $206 \mathrm{p}$.

90. Sanmiguel R, Rodriguez, C, Aguirre $W$. 2015. Sustancias húmicas en la disminución del estrés en gallinas ponedoras durante la fase posmuda. Revista Cienc Agric 12(1): 59-66.

91. Sanmiguel R, Rondón I. 2014. Suplementación con sustancias húmicas en gallinas ponedoras durante la fase posmuda. Rev CES Med Vet Zootec 9: 169-178.
92. Sans E, Federici J, Dahlke F, Molento C. 2014. Evaluation of free-range broilers using the Welfare Quality ${ }^{\circledR}$ Protocol. Rev Bras Sci Avic 16: 297-306. doi: 10.1590/1516-635x1603297-306

93. Schmidt M, Lahrmann K, Ammon C, Berg W, Schon P, Hoffmann G. 2013. Assessment of body temperature in sows by tow infrared thermography methods at various body surface locations. J Swine Health Prod 21: 203-209.

94. Selye H. 1946. The general adaptation syndrome and the diseases of adaptation. J Clin Endocrinol Metabol 6: 117-230. doi: 10.1210/jcem-6-2-117

95. Sharma S, Ramesh K, Hyder I, Uniyal $S$, Yadav VP, Panda, RP, Maurya VP, et al. 2013. Effect of melatonin administration on thyroid hormones, cortisol and expression profile of heat shock proteins in goats (Capra hircus) exposed to heat stress. Small Rumin Res 112:216-223. doi: 10.1016/j.smallrumres.2012.12.008

96. Sheriff M, Krebs C, Boonstra R. 2010. Assessing stress in animal populations: Do fecal and plasma glucocorticoids tell the same story? Gen Comp Endocrinol 166: 614-619. doi: 10.1016/j.ygcen.2009.12.017

97. Shini S, Kaiser P, Shini A, Bryden W. 2008. Biological response of chickens (Gallus gallus domesticus) induced by corticosterone and a bacterial endotoxin. Comp Biochem Physiol B Biochem Mol Biol 149: 324-333. doi: 10.1016/ j.cbpb.2007.10.003

98. Singh $R$, Cook $N$, Cheng $M$, Silversides $F$. 2009. Invasive and noninvasive measurement of stress in laying hens kept in conventional cages and in floor pens. Poult Sci $88: 1346-1351$. doi: $10.3382 /$ ps.2008-00300

99. Sordillo L, Aitken S. 2008. Impact of oxidative stress on the health and immune function of dairy cattle. Vet Immunol Immunopathol 128: 104-109. doi: 10.1016/j.vetimm.2008.10.305 
100.Star L, Kemp B, Van der Anker I, Parmentier H. 2008. Effect of single or combined climatic and hygienic stress on natural and specific humoral immune competence in four layer lines. Poultr Sci 86: 1894-1903.

101.Stewart M, Stafford K, Dowling S, Schaefer A, Webster J. 2008. Eye temperature and heart rate variability of calves disbudded with or without local anaesthetic. Physiol Behav 93: 789-797. doi: 10.1016/j.physbeh.2007.11.044

102.Stubsjoen S, Bohlin J, Dahl E, Knappe-Poindecker M, Fjeldaas T, Lepschy M, et al. 2015a. Assessment of chronic stress in sheep (part I): the use of cortisol and cortisone in hair as non-invasive biological markers. Small Rumin Res 132: 25-31. doi: 10.1016/ j.smallrumres.-2015.09.015

103.Stubsjoen S, Bohlin J, Dahl E, Knappe-Poindecker M, Fjeldaas T, Lepschy M, Palme R, Langbein J, Ropstad E. 2015b. Assessment of chronic stress in sheep (part II): exploring heart rate variability as a non-invasive measure to evaluate cardiac regulation. Small Rumin Res 133: 30-35. doi: 10.1016/j.smallrumres.-2015.10.026

104.Tarvainen M, Niskanen J, Lipponen J, Ranta-aho P, Karjalainen P. 2014. Kubios HRV - Heart rate variability analysis software. Comp Meth Prog Biomed 113: 210-220. doi: 10.1016/ j.cmpb.2013.-07.024

105. Toledo L, Vargas V. 2016. Bienestar animal y calidad de la carne. En: Martínez R (ed). Bioética, inocuidad y bienestar animal. México: Univ. de Juanajuato. p 1-24.
106. Vermeulen L, Van de Perre V, Permentier L, De Bie S, Verbeke G, Geers R. 2015. Pre-slaughter handling and pork quality. Meat Sci 100: 118-123. doi: 10.1016/j.meatsci.2014.09.148

107.Valance D, Desprès G, Boissy A, Mignon-Grasteau S, Constantin P, Leterrier C. 2007. Genetic selection on a behavioral fear trait is associated with changes in heart rate variability in quail. Genes Brain Behav 6: 339-346. doi: 10.1111/j.1601-183X.2006.00262.x

108.Wang C, King W, Woods LC. 2004. Physiological indicators of divergent stress responsiveness in male striped bass broodstock. Aquaculture 232: 665678. doi: 10.1016/j.aquaculture.2003.08.008

109.Wang S, Ni Y, Guo F, Fu W, Grossman $\boldsymbol{R}$, Zhao R. 2013. Effect on corticosterone on growth and welfare on broiler chickens showing long or short tonic immobility. Comp Biochem Physiol A Mol Integr Physiol 164: 537-543. doi: 10.1016/j.cbpa.2012.12.014

110. Whay H, Main D, Green L, Heaven G, Howell H, Morgan M, Pearson A, Webster A. 2007. Assessment of the behavior and welfare of laying hens on free range units. Vet Rec 161: 119-128.

111.Webster A. 2004. Welfare implications of avian osteoporosis. Poult Sci 83: 184-192.

112. Welfare Quality. 2009. Principios y criterios para el bienestar de los animales de granja. Internet]. Disponible en: http:/ /www.welfarequality.net/downloadattachment/41858/19877/ WQ\%20_\%20-Factsheet_10_$07 \_s p \% 20(2) \cdot p d f$ 